\title{
On The Grand Wiener Amalgam Spaces
}

\author{
A.Turan Gürkanlı
}

\begin{abstract}
In this article, notations are included in Section 1. In Section 2, we define the grand Wiener amalgam space by using the classical Wiener amalgam space $[9,15,16,17]$ and the generalized grand Lebesgue space $[18,13]$. Section 3 , concerns the inclusions between these spaces and some applications. In last section Section 4, we prove the Hölder's inequality for grand Wiener amalgam space. We also find the associate space and dual of this space, and we prove that the grand Wiener amalgam space is not reflexive.
\end{abstract}

\section{Notations}

Let $\Omega$ be locally compact hausdorff space and let $(\Omega, \mathfrak{B}, \mu)$ be finite Borel measure space. The grand Lebesgue space $L^{p)}(\mu)$ was introduced in [18] by the norm

$$
\|f\|_{p)}=\sup _{0<\varepsilon \leq p-1}\left(\varepsilon \int_{\Omega}|f|^{p-\varepsilon} d \mu\right)^{\frac{1}{p-\varepsilon}}
$$

where $1<p<\infty$. This is a Banach space. For $0<\varepsilon \leq p-1, L^{p}(\mu) \subset L^{p)}(\mu) \subset$ $L^{p-\varepsilon}(\mu)$ hold. For some properties and applications of $L^{p)}(\mu)$ spaces we refer to papers [1] , [4] , [6], [7], [12] and [13] . A generalization of the grand Lebesgue spaces are the spaces $L^{p), \theta}(\mu), \theta \geq 0$, defined by the norm (see [1], [13])

$$
\|f\|_{p), \theta, \mu}=\|f\|_{p), \theta}=\sup _{0<\varepsilon \leq p-1} \varepsilon^{\frac{\theta}{p-\varepsilon}}\left(\int_{\Omega}|f|^{p-\varepsilon} d \mu\right)^{\frac{1}{p-\varepsilon}}=\sup _{0<\varepsilon \leq p-1} \varepsilon^{\frac{\theta}{p-\varepsilon}}\|f\|_{p-\varepsilon} ;
$$

when $\theta=0$ the space $L^{p), 0}(\mu)$ reduces to Lebesgue space $L^{p}(\mu)$ and when $\theta=1$ the space $L^{p), 1}(\mu)$ reduces to grand Lebesgue space $L^{p)}(\mu)$. We have for all $1<p<\infty$ and $\varepsilon>0$

$$
L^{p}(\mu) \subset L^{p), \theta}(\mu) \subset L^{p-\varepsilon}(\mu) .
$$

Different properties and applications of these spaces were discussed in [1], [13] and [4].

2000 Mathematics Subject Classification. Primary 46E30; Secondary 46E35; 46B70.

Key words and phrases. Grand Lebesgue space, generalized grand Lebesgue space, grand Wiener Amalgam space. 
Let $1 \leq p<\infty, \theta \geq 0$, and $J$ be the one of the set of $\mathbb{N}, \mathbb{Z}$ or $\mathbb{Z}^{n}$. We define the grand Lebesgue sequence space $\ell^{p), \theta}=\ell^{p), \theta}(J)$ by the norm

$$
\|u\|_{\ell^{p), \theta}(J)}=\sup _{0<\varepsilon \leq p-1}\left(\varepsilon^{\theta} \sum_{k \in J}\left|u_{k}\right|^{p-\varepsilon}\right)^{\frac{1}{p-\varepsilon}}=\sup _{0<\varepsilon \leq p-1} \varepsilon^{\frac{\theta}{p-\varepsilon}}\|u\|_{\ell^{p-\varepsilon}(J)} .
$$

Let $p^{\prime}=\frac{p}{p-1}, 1<p<\infty$. First consider an auxiliary space namely $L^{\left(p^{\prime}, \theta\right.}(\Omega), \theta>$ 0 , defined by

$$
\|g\|_{\left(p^{\prime}, \theta\right.}=\inf _{g=\sum_{k=1}^{\infty} g_{k}}\left\{\sum_{k=1}^{\infty} \inf _{0<\varepsilon \leq p-1} \varepsilon^{-\frac{\theta}{p-\varepsilon}}\left(\int_{\Omega}\left|g_{k}\right|^{(p-\varepsilon)^{\prime}} d x\right)^{\frac{1}{(p-\varepsilon)^{\prime}}}\right\}
$$

where the functions $g_{k}, k \in \mathbb{N}$, being in $\mathcal{M}_{0}$, the set of all real valued measurable functions, finite a.e. in $\Omega$. After this definition the generalized small Lebesgue spaces have been defined by

$$
L^{p)^{\prime}, \theta}(\Omega)=\left\{g \in \mathcal{M}_{0}:\|g\|_{p)^{\prime}, \theta}<+\infty\right\},
$$

where

$$
\|g\|_{p)^{\prime}, \theta}=\sup _{\substack{0 \leq \psi \leq|g| \\ \psi \in L^{\left(p^{\prime}, \theta\right.}}}\|\psi\|_{\left(p^{\prime}, \theta\right.}
$$

For $\theta=0$ it is $\|f\|_{\left(p^{\prime}, 0\right.}=\|f\|_{p^{\prime}, \theta},[5],[11]$.

Let $1 \leq p, q \leq \infty$. The space $\left(L^{p)}\right)_{l o c}$ consists of (classes of) measurable functions $f: \Omega \rightarrow \mathbb{C}$ such that $f \chi_{K} \in L^{p)}$, for any compact subset $K \subset \Omega$, where $\chi_{K}$ is the characteristic function of $f$. It is a topological vector space with the family of seminorms $f \rightarrow\|f\|_{p)}$. Since $L^{p} \subset L^{p)}$, it is easy to show that $\left(L^{p}\right)_{l o c} \hookrightarrow\left(L^{p)}\right)_{l o c}$.

\section{The Grand Wiener Amalgam Space}

Definition 1. Let $\Omega$ be locally compact hausdorff space and let $(\Omega, \mathfrak{B}, \mu)$ be finite Borel measure space. Also assume that $1<p, q<\infty$ and $Q \subset \Omega$ is a fix compact subset with nonemty interior. The grand Wiener amalgam space $W\left(L^{p), \theta_{1}}, L^{q), \theta_{2}}\right)$ consists of all functions (classes of) $f \in\left(L^{p), \theta_{1}}\right)_{l o c}$ such that the control function

$$
F_{f}^{p), \theta_{1}}(x)=F_{f}^{p), \theta_{1}, Q}(x)=\left\|f \cdot \chi_{Q+x}\right\|_{p), \theta_{1}}=\sup _{0<\varepsilon \leq p-1} \varepsilon^{\frac{\theta_{1}}{p-\varepsilon}}\left\|f \cdot \chi_{Q+x}\right\|_{p-\varepsilon}
$$

lies in $L^{q), \theta_{2}}$. The norm of $W\left(L^{p), \theta_{1}}, L^{q), \theta_{2}}\right)$ defined by

$$
\|f\|_{W\left(L^{\left.p), \theta_{1}, L^{q), \theta_{2}}\right)}\right.}=\left\|F_{f}^{p), \theta_{1}}\right\|_{q), \theta_{2}}=\|\| f \cdot \chi_{Q+x}\left\|_{p), \theta_{1}}\right\|_{q), \theta_{2}} .
$$

Since generalized grand Lebesgue spaces are not translation invariant, this result reflects to the grand Wiener amalgam spaces. Then the definition of $W\left(L^{p), \theta_{1}}, L^{q), \theta_{2}}\right)$ is depend of the choice of $Q$. We give the following theorem for the independedness.

THEOREM 1. if $\theta_{2}=0$ then the definition of grand Wiener amalgam space $W\left(L^{p), \theta_{1}}, L^{q), \theta_{2}}\right)$ is independent of the choice of $Q$, i.e., different choices of $Q$ define the same space with equivalent norms. 
Proof. We know that when $\theta_{2}=0$, the generalized grand Lebesgue space reduces to lebesgue space $L^{p}(\Omega)$. Then if $\theta_{2}=0$, the grand Wiener amalgam space $W\left(L^{p), \theta_{1}}, L^{q), \theta_{2}}\right)$ reduces to $W\left(L^{p), \theta_{1}}, L^{q}\right)$. Since $L^{q}(\Omega)$ is solid and strongly translation invariant, by using the proof technic in Proposition 11.3.2 (b) in [15] or Theorem 2 in [9], the proof is completed.

Theorem 2. Let $1<p, q<\infty$, and $\theta \geq 0$. Then $W\left(L^{p), \theta}, L^{q), \theta}\right)$ is a Banach function space (shortly BF-space), namely its norm satisfies the following properties, where $f, g$ and $f_{n}$ are in $W\left(L^{p), \theta}, L^{q), \theta}\right), \lambda \geq 0$ and $E$ is a measurable subset of $\Omega$ :

1. $\|f\|_{W\left(L^{p), \theta}, L^{q), \theta}\right)} \geq 0$

2. $\|f\|_{W\left(L^{p), \theta}, L^{q), \theta}\right)}=0$ if and only if $f=0$ a.e in $\Omega$

3. $\|\lambda f\|_{W\left(L^{p), \theta}, L^{q), \theta}\right)}=\lambda\|f\|_{W\left(L^{p), \theta}, L^{q), \theta}\right)}$

4. $\left.\|f+g\|_{W\left(L^{p), \theta}, L^{q), \theta}\right)} \leq\|f\|_{W\left(L^{p), \theta}, L^{q), \theta}\right)}+\|g\|_{W\left(L^{p), \theta}, L^{q), \theta}\right.}\right)$

5. if $|g| \leq|f|$ a.e. in $\Omega$, then $\|g\|_{W\left(L^{p), \theta}, L^{q), \theta}\right)} \leq\|f\|_{W\left(L^{p), \theta}, L^{q), \theta}\right)}$

6. if $0 \leq f_{n} \uparrow f$ a.e. in $\Omega$, then $\left\|f_{n}\right\|_{W\left(L^{p), \theta}, L^{q), \theta}\right)} \uparrow\|f\|_{W\left(L^{p), \theta}, L^{q), \theta}\right)}$

7. $\left\|\chi_{E}\right\|_{W\left(L^{p), \theta}, L^{q), \theta}\right)}<+\infty$

8. $\int_{E}|f| d x \leq C(p, \theta, E)\|f\|_{W\left(L^{p), \theta}, L^{q), \theta}\right)}$

for some $0<C<\infty$.

Proof. The first three properties follow from the definition of the norm $\left.\|\cdot\|_{W\left(L^{p), \theta}, L^{q), \theta}\right.}\right)$.

Proof of property 4 . Let $f, g \in W\left(L^{p), \theta}, L^{q), \theta}\right)$. Then

$$
\begin{aligned}
\|f+g\|_{W\left(L^{p), \theta}, L^{q), \theta}\right)} & =\|\|(f+g) \chi_{Q+x}\left\|_{p), \theta}\right\|_{q), \theta}=\|\| f \chi_{Q+x}+g \chi_{Q+x}\left\|_{p), \theta}\right\|_{q), \theta} \\
& \leq\|\| f \chi_{Q+x}\left\|_{p), \theta}+\right\| g \chi_{Q+x}\left\|_{p), \theta}\right\|_{q), \theta}=\left\|F_{f}^{p), \theta}(x)+F_{g}^{p), \theta}(x)\right\|_{q), \theta} \\
& \leq\left\|F_{f}^{p), \theta}(x)\right\|_{q), \theta}+\left\|F_{g}^{p), \theta}(x)\right\|_{q), \theta}=\|f\|_{W\left(L^{p), \theta}, L^{q), \theta}\right)}+\|g\|_{W\left(L^{p), \theta}, L^{q), \theta}\right)} .
\end{aligned}
$$

Proof of property 5. Let $|g| \leq|f|$. Then $|g| \chi_{Q+x} \leq|f| \chi_{Q+x}$ a.e. in $\Omega$. Since $L^{p), \theta}(\Omega)$ is a BF-space on $\Omega$, we have

$$
F_{g}^{p), \theta}(x)=\left\|g \chi_{Q+x}\right\|_{p), \theta} \leq\left\|f \chi_{Q+x}\right\|_{p), \theta}=F_{f}^{p), \theta}(x) .
$$

Thus we obtain $\|g\|_{W\left(L^{p), \theta}, L^{q), \theta}\right)} \leq\|f\|_{W\left(L^{p), \theta}, L^{q), \theta}\right)}$.

Proof of property 6 . Since $0 \leq f_{n} \uparrow f$ a.e. in $\Omega$, then $f_{n} \chi_{Q+x} \uparrow f \chi_{Q+x}$ a.e. in $\Omega$. Then

$$
F_{f_{n}}^{p), \theta}(x)=\left\|f_{n} \chi_{Q+x}\right\|_{p), \theta} \uparrow\left\|f \chi_{Q+x}\right\|_{p), \theta}=F_{f}^{p), \theta}(x)
$$

by proposition 2.1, in [1] . One more applaying this proposition we have

$$
\left\|f_{n}\right\|_{W\left(L^{p), \theta}, L^{q), \theta}\right)}=\|\| f_{n} \chi_{Q+x}\left\|_{p), \theta}\right\|_{q), \theta} \uparrow\|\| f \chi_{Q+x}\left\|_{p), \theta}\right\|_{q), \theta}=\|f\|_{W\left(L^{p), \theta}, L^{q), \theta}\right)} .
$$


Proof of property 7 . Since $\varepsilon^{\frac{\theta}{p-\varepsilon}}$ is increasing in $[0, p-1]$, and $\mu(\Omega)$ is finite, then

$$
\begin{aligned}
\left\|\chi_{E} \chi_{Q+x}\right\|_{W\left(L^{p), \theta}, L^{q), \theta}\right)} & =\left\|\chi_{E \cap Q+x}\right\|_{W\left(L^{p), \theta}, L^{q), \theta}\right)}=\|\| \chi_{E \cap Q+x}\left\|_{p), \theta}\right\|_{q), \theta}(1) \\
& =\left\|\sup _{0<\varepsilon \leq p-1} \varepsilon^{\frac{\theta}{p-\varepsilon}}\right\| \chi_{E \cap Q+x}\left\|_{p-\varepsilon}\right\|_{q), \theta} \\
& =\left\|\sup _{0<\varepsilon \leq p-1} \varepsilon^{\frac{\theta}{p-\varepsilon}}\left\{\int_{\Omega}\left|\chi_{E \cap Q+x}\right|^{\frac{1}{p-\varepsilon}} d \mu\right\}^{\frac{1}{p-\varepsilon}}\right\| \|_{q), \theta} \\
& \leq\left\|\sup _{0<\varepsilon \leq p-1} \varepsilon^{\frac{\theta}{p-\varepsilon}}[\mu(\Omega)]^{\frac{1}{p-\varepsilon}}\right\|_{q), \theta} .
\end{aligned}
$$

If $\mu(\Omega)<1$, then $\mu(\Omega)^{\frac{1}{p-\varepsilon}}<\mu(\Omega)^{\frac{1}{p}}$ for all $0<\varepsilon \leq p-1$. Since $\varepsilon^{\frac{\theta}{p-\varepsilon}}$ is increasing, by (1) we have

$$
\begin{aligned}
\left\|\chi_{E} \chi_{Q+x}\right\|_{W\left(L^{p), \theta}, L^{q), \theta}\right)} & \leq\left\|\sup _{0<\varepsilon \leq p-1} \varepsilon^{\frac{\theta}{p-\varepsilon}}[\mu(\Omega)]^{\frac{1}{p-\varepsilon}}\right\|_{q), \theta} \\
& \leq\left\|(p-1)^{\theta}[\mu(\Omega)]^{\frac{1}{p}}\right\|_{q), \theta} \\
& =\sup _{0<\eta \leq q-1} \eta^{\frac{\theta}{q-\eta}}\left\|(p-1)^{\theta}[\mu(\Omega)]^{\frac{1}{p}}\right\|_{q-\eta} \\
& =(p-1)^{\theta}(q-1)^{\theta}[\mu(\Omega)]^{\frac{1}{p}}[\mu(\Omega)]^{\frac{1}{q}} \\
& =(p-1)^{\theta}(q-1)^{\theta}[\mu(\Omega)]^{\frac{1}{p}+\frac{1}{q}}<\infty .
\end{aligned}
$$

If $\mu(\Omega)>1$, then $\mu(\Omega)^{\frac{1}{p-\varepsilon}}>\mu(\Omega)^{\frac{1}{p}}$ for all $0<\varepsilon \leq p-1$. Again from (1), we have

$$
\begin{aligned}
\left\|\chi_{E} \chi_{Q+x}\right\|_{W\left(L^{p), \theta}, L^{q), \theta}\right)} & \leq\left\|\sup _{0<\varepsilon \leq p-1} \varepsilon^{\frac{\theta}{p-\varepsilon}}[\mu(\Omega)]^{\frac{1}{p-\varepsilon}}\right\|_{q), \theta} \\
& =\left\|(p-1)^{\theta}[\mu(\Omega)]\right\|_{q), \theta}=\sup _{0<\eta \leq q-1} \eta^{\frac{\theta}{q-\eta}}\left\|(p-1)^{\theta} \mu(\Omega)\right\|_{q-\eta} \\
& \leq(p-1)^{\theta}(q-1)^{\theta}[\mu(\Omega)]^{2}<\infty .
\end{aligned}
$$

Proof of property 8 . Fix $0<\varepsilon \leq p-1$. By the Hölder's inequality for Wiener's amalgam space we have

$\int_{E}|f| d x=\int_{\Omega}\left|f \chi_{E}\right| d x \leq\|f\|_{W\left(L^{p-\varepsilon}, L^{q-\varepsilon}\right)}\left\|\chi_{E}\right\|_{W\left(L^{\left.(p-\varepsilon)^{\prime}, L^{(q-\varepsilon)^{\prime}}\right)}\right.}=C\|f\|_{W\left(L^{\left.p-\varepsilon, L^{q-\varepsilon}\right)}\right.}$

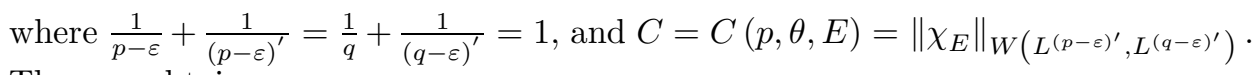
Thus we obtain

$$
\int_{E}|f| d x \leq C \sup _{0<\varepsilon \leq p-1} \varepsilon^{\frac{\theta}{p-\varepsilon}}\|f\|_{W\left(L^{p-\varepsilon}, L^{q-\varepsilon}\right)}=C\|f\|_{W\left(L^{p)}, L^{q)}\right)} .
$$

TheOREM 3. The grand Wiener amalgam space $W\left(L^{p), \theta}, L^{q), \theta}\right)$ is a Banach space. 
Proof. To show that $W\left(L^{p), \theta}, L^{q), \theta}\right)$ is a Banach space it is suffices to show that if $\left(f_{n}\right)_{n \in \mathbb{N}}$ is a sequence in $W\left(L^{p), \theta}, L^{q), \theta}\right)$ with

$$
\sum_{n \in \mathbb{N}}\left\|f_{n}\right\|_{W\left(L^{p), \theta}, L^{q), \theta}\right)}<\infty
$$

then $\sum_{n \in \mathbb{N}} f_{n}$ converges to an element of $W\left(L^{p), \theta}, L^{q), \theta}\right)$. The proof of this is mutadis and mutandis same as in the proof of completness of Wiener amalgam space $W\left(L^{p}, L^{q}\right)$, (see Proposition 11.3.2, in [15], and [9]).

\section{Inclusions and consequences}

Proposition 1. Let $1<p, q<\infty$. Then for an arbitrary $\varepsilon$ and $\eta, 0<\varepsilon \leq$ $p-1,0<\eta \leq q-1$, we have

$$
W\left(L^{p}, L^{q}\right) \subset W\left(L^{p), \theta}, L^{q), \theta}\right) \subset W\left(L^{p-\varepsilon}, L^{q-\eta}\right) .
$$

If $q \leq p$, then the inclusion $W\left(L^{p}, L^{q}\right) \subset W\left(L^{p), \theta}, L^{q), \theta}\right)$ is strict.

Proof. By the definition of generalized grand Lebesgue space we have

$$
F_{f}^{p-\varepsilon}(x)=\left\|f \cdot \chi_{Q+x}\right\|_{p-\varepsilon} \leq\left\|f \cdot \chi_{Q+x}\right\|_{p), \theta}=F_{f}^{p), \theta}(x) .
$$

Then from (2),

$$
\begin{aligned}
\|f\|_{W\left(L^{p-\varepsilon}, L^{q-\eta}\right)} & =\|\| f \cdot \chi_{Q+x}\left\|_{p-\varepsilon}\right\|_{q-\eta} \leq\left\|F_{f}^{p), \theta}(x)\right\|_{q-\eta} \\
& \leq \sup _{0<\eta \leq q-1} \varepsilon^{\frac{\theta}{q-\eta}}\left\|F_{f}^{p), \theta}(x)\right\|_{q), \theta}=\|f\|_{W\left(L^{p), \theta}, L^{q, \theta)}\right)} .
\end{aligned}
$$

This implies $W\left(L^{p), \theta}, L^{q), \theta}\right) \subset W\left(L^{p-\varepsilon}, L^{q-\eta}\right)$.

We now want to show that $W\left(L^{p}, L^{q}\right) \subset W\left(L^{p), \theta}, L^{q), \theta}\right)$. It is known that $L^{p} \subset L^{p), \theta}$ and $L^{q} \subset L^{q), \theta}$. Then there exists $D_{1}>0$ and $D_{2}>0$ such that

$$
\begin{gathered}
\|g\|_{p), \theta} \leq D_{1}\|g\|_{p}, \\
\|h\|_{q), \theta} \leq D_{2}\|h\|_{q} .
\end{gathered}
$$

Then by (2) and (3)

$$
F_{f}^{p), \theta}(x)=\left\|f \cdot \chi_{Q+x}\right\|_{p), \theta} \leq D_{1}\left\|f \cdot \chi_{Q+x}\right\|_{p}=D_{1} F_{f}^{p}(x) .
$$

Hence by (3) and (4),

$$
\|f\|_{W\left(L^{p), \theta}, L^{q), \theta}\right)}=\left\|F_{f}^{p), \theta}\right\|_{q), \theta} \leq\left\|D_{1} F_{f}^{p}\right\|_{q), \theta} \leq D_{1} D_{2}\left\|F_{f}^{p}\right\|_{q}=\|f\|_{W\left(L^{p}, L^{q}\right)} .
$$

Thus $W\left(L^{p}, L^{q}\right) \subseteq W\left(L^{p), \theta}, L^{q), \theta}\right)$. 
To see that the inclusion $W\left(L^{p}, L^{q}\right) \subseteq W\left(L^{p), \theta}, L^{q), \theta}\right)$ is strict, take $\Omega=$ $(0,1)$ and $f(x)=x^{-\frac{1}{p}}$, for $p>1$. Then

$$
\begin{aligned}
F_{f}^{p)}(x) & =\left\|t^{-\frac{1}{p}} \chi_{Q+x}\right\|_{p), \theta} \leq\left\|t^{-\frac{1}{p}}\right\|_{p), \theta}=\sup _{0<\varepsilon \leq p-1} \varepsilon^{\frac{\theta}{p-\varepsilon}}\left(\left\|t^{-\frac{1}{p}}\right\|_{p-\varepsilon}\right) \\
& =\sup _{0<\varepsilon \leq p-1} \varepsilon^{\frac{\theta}{p-\varepsilon}}\left(\int_{0}^{1} t^{-\frac{1}{p}(p-\varepsilon)} d t\right)^{\frac{1}{p-\varepsilon}}=\sup _{0<\varepsilon \leq p-1} \varepsilon^{\frac{\theta}{p-\varepsilon}}\left(\lim _{a \rightarrow 0}\left(\int_{a}^{1} t^{-\frac{1}{p}(p-\varepsilon)} d t\right)^{\frac{1}{p-\varepsilon}}\right) \\
& =\sup _{0<\varepsilon \leq p-1} \varepsilon^{\frac{\theta}{p-\varepsilon}}\left[\lim _{a \rightarrow 0}\left(\left.\frac{p}{\varepsilon} t^{\frac{\varepsilon}{p}}\right|_{t=a} ^{t=1}\right)\right]^{\frac{1}{p-\varepsilon}}=\sup _{0<\varepsilon \leq p-1} \varepsilon^{\frac{\theta}{p-\varepsilon}}\left[\lim _{a \rightarrow 0}\left(\frac{p}{\varepsilon}\left(1-a^{\frac{\varepsilon}{p}}\right)\right)\right]^{\frac{1}{p-\varepsilon}} \\
& =\sup _{0<\varepsilon \leq p-1} \varepsilon^{\frac{\theta}{p-\varepsilon}}\left(\frac{p}{\varepsilon}\right)^{\frac{1}{p-\varepsilon}}=\sup _{0<\varepsilon \leq p-1} \varepsilon^{\frac{\theta-1}{p-\varepsilon}} \cdot p^{\frac{1}{p-\varepsilon}} .
\end{aligned}
$$

Since $\theta \geq 1$,

$$
F_{f}^{p)}(x)=\sup _{0<\varepsilon \leq p-1} \varepsilon^{\frac{\theta-1}{p-\varepsilon}} \cdot p^{\frac{1}{p-\varepsilon}} \leq(p-1)^{\theta-1} p<p^{\theta} .
$$

Thus from (5),

$$
\begin{aligned}
\|f\|_{W\left(L^{p)}, L^{q)}\right)} & =\left\|F_{f}^{p)}\right\|_{q), \theta}<\left\|p^{\theta}\right\|_{q), \theta} \\
& =\sup _{0<\eta \leq q-1} \eta^{\frac{\theta}{q-\eta}}\left\{\int_{0}^{1}\left(p^{\theta}\right)^{q-\eta} d x\right\}^{\frac{1}{q-\eta}}=\sup _{0<\eta \leq q-1} \varepsilon^{\frac{\theta}{q-\eta}} p^{\theta} \\
& =(q-1)^{\theta} p^{\theta}<\infty .
\end{aligned}
$$

Then $f(x)=x^{-\frac{1}{p}} \in W\left(L^{p), \theta}, L^{q), \theta}\right)(0,1)$. In the other hand it is easy to show that $f(t)=t^{-\frac{1}{p}} \notin L^{p}(0,1)$. Since $W\left(L^{p}, L^{q}\right)(0,1) \subset L^{p}(0,1)$ for $q \leq p$, then $f(t)=$ $t^{-\frac{1}{t}} \notin W\left(L^{p}, L^{q}\right)(0,1)$. So we have $f(t)=t^{-\frac{1}{p}} \in W\left(L^{p), \theta}, L^{q)}, \theta\right) \backslash W\left(L^{p}, L^{q}\right)$. Thus if $q \leq p$, the inclusion $W\left(L^{p}, L^{q}\right) \subset W\left(L^{p), \theta}, L^{q), \theta}\right)$ is strict.

Proposition 2. If $1<p_{2} \leq p_{1}<\infty$ and $1<q<\infty$, then $W\left(L^{\left.p_{1}\right), \theta}, L^{q), \theta}\right) \subset$ $W\left(L^{\left.p_{2}\right), \theta}, L^{q), \theta}\right)$.

Proof. Let $f \in W\left(L^{\left.p_{1}\right), \theta}, L^{q), \theta}\right)$. Then $f \chi_{Q+x} \in L^{\left.p_{1}\right), \theta}$. Since $p_{2} \leq p_{1}$, by Theorem 3, in [15] we have $L^{\left.p_{1}\right), \theta} \subset L^{\left.p_{2}\right), \theta}$, and

$$
\left\|f \chi_{Q+x}\right\|_{\left.p_{2}\right), \theta} \leq C\left\|f \chi_{Q+x}\right\|_{\left.p_{1}\right), \theta}, x \in \Omega
$$

for some $C>0$. Thus by the solidness of $L^{q), \theta}$,

$$
\begin{aligned}
\|f\|_{W\left(L^{\left.p_{2}\right), \theta}, L^{q), \theta}\right)} & =\|\| f \cdot \chi_{Q+x}\left\|_{\left.p_{2}\right), \theta}\right\|_{\|f\|_{q), \theta}} \\
& \leq C\|\| f \cdot \chi_{Q+x}\left\|_{\left.p_{1}\right), \theta}\right\|_{q), \theta}=\|f\|_{W\left(L^{\left.p_{1}\right), \theta}, L^{q), \theta}\right)},
\end{aligned}
$$

and we have $W\left(L^{\left.p_{1}\right), \theta}, L^{q), \theta}\right) \subset W\left(L^{\left.p_{2}\right), \theta}, L^{q), \theta}\right)$.

Proposition 3. If $1<q_{2} \leq q_{1}<\infty$ and $1<p<\infty$, then $W\left(L^{p), \theta}, L^{\left.q_{1}\right), \theta}\right) \subset$ $W\left(L^{p), \theta}, L^{\left.\left.q_{2}\right), \theta\right)}\right)$. 
Proof. Let $f \in W\left(L^{p), \theta}, L^{\left.q_{1}\right), \theta}\right)$. Then $F_{f}^{p)} \in L^{\left.q_{1}\right), \theta}$. Since $1<q_{2} \leq q_{1}<$ $\infty$,by Theorem 3 in [14], there exists $C>0$ such that

$$
\left\|F_{f}^{p), \theta}\right\|_{W\left(L^{p), \theta}, L^{\left.q_{2}\right), \theta}\right)} \leq C\left\|F_{f}^{p), \theta}\right\|_{W\left(L^{p), \theta}, L^{\left.q_{1}\right), \theta}\right)}<\infty
$$

for all $\theta>0$. Then $F_{f}^{p), \theta} \in L^{\left.q_{2}\right), \theta}$. This implies $f \in W\left(L^{p), \theta}, L^{\left.q_{2}\right), \theta}\right)$. Thus we have the inclusion

$$
W\left(L^{p), \theta}, L^{\left.q_{1}\right), \theta}\right) \subset W\left(L^{p), \theta}, L^{\left.\left.q_{2}\right), \theta\right)}\right) .
$$

By using the Proposition 2 and proposition 3, we easily prove the following corollary.

Corollary 1. If $1<p_{2} \leq p_{1}<\infty$ and $1<q_{2} \leq q_{1}<\infty$, then $W\left(L^{\left.p_{1}\right), \theta}, L^{\left.q_{1}\right), \theta}\right) \subset$ $W\left(L^{\left.p_{2}\right), \theta}, L^{\left.q_{2}\right), \theta}\right)$.

Proposition 4. Let $1<p_{i}, q_{i}<\infty,(i=1,2,3)$. If there exist constants $C_{1}>$ $0, C_{1}>0$, such that for all $u \in L^{\left.p_{1}\right), \theta}, v \in L^{\left.p_{2}\right)}$,

$$
\|u v\|_{L^{\left.p_{3}\right), \theta}} \leq C_{1}\|u\|_{L^{\left.p_{1}\right), \theta}}\|v\|_{L^{\left.p_{2}\right), \theta}}
$$

and for all $u \in L^{\left.q_{1}\right), \theta}, v \in L^{\left.q_{2}\right), \theta}$

$$
\|u v\|_{L^{\left.q_{3}\right), \theta}} \leq C_{2}\|u\|_{L^{\left.q_{1}\right), \theta}}\|v\|_{L^{\left.q_{2}\right), \theta}},
$$

then there exists a constant $C>0$, such that for all $f \in W\left(L^{\left.p_{1}\right), \theta}, L^{\left.q_{1}\right), \theta}\right)$ and $g \in W\left(L^{\left.p_{2}\right), \theta}, L^{\left.q_{2}\right), \theta}\right)$, we have $f g \in W\left(L^{\left.p_{3}\right), \theta}, L^{\left.q_{3}\right), \theta}\right)$ and

$$
\|f . g\|_{W\left(L^{\left.p_{3}\right), \theta}, L^{\left.q_{3}\right), \theta}\right)} \leq C_{1} C_{2}\|f\|_{W\left(L^{\left.p_{1}\right), \theta}, L^{\left.q_{1}\right), \theta}\right)}\|g\|_{W\left(L^{\left.p_{2}\right), \theta}, L^{\left.q_{2}\right), \theta}\right)} \cdot
$$

Proof. Let $f \in W\left(L^{\left.p_{1}\right), \theta}, L^{\left.q_{1}\right), \theta}\right)$ and $g \in W\left(L^{\left.p_{2}\right), \theta}, L^{\left.q_{2}\right), \theta}\right)$. Since $\chi_{Q+x}^{2}=$ $\chi_{Q+x}$, from (7) and (8),

$$
\begin{aligned}
\|f g\|_{W\left(L^{\left.p_{3}\right), \theta}, L^{\left.q_{3}\right), \theta}\right.} & =\|\|(f g) \chi_{Q+x}\left\|_{L^{\left.p_{3}\right), \theta}}\right\|_{L^{\left.q_{3}\right), \theta}} \\
& =\|\|\left(f \chi_{Q+x}\right)\left(f \chi_{Q+x}\right)\left\|_{L^{\left.p_{3}\right), \theta}}\right\|_{L^{\left.q_{3}\right), \theta}} \\
& \leq C_{1}\|\| f \chi_{Q+x}\left\|_{L^{\left.p_{1}\right), \theta}}\right\| f \chi_{Q+x}\left\|_{L^{\left.p_{2}\right), \theta}}\right\|_{L^{\left.q_{3}\right), \theta}} \\
& \leq C_{1} C_{2}\|\| f \chi_{Q+x}\left\|_{L^{\left.p_{1}\right), \theta}}\right\|_{L^{\left.q_{1}\right), \theta}}\|\| f \chi_{Q+x}\left\|_{L^{\left.p_{2}\right), \theta}}\right\|_{L^{\left.q_{2}\right), \theta}} \\
& =C_{1} C_{2}\|f\|_{W\left(L^{\left.p_{1}\right), \theta}, L^{\left.q_{1}\right), \theta}\right)}\|g\|_{W\left(L^{\left.p_{2}\right), \theta}, L^{\left.q_{2}\right), \theta}\right.} .
\end{aligned}
$$

Proposition 5. Let $1<p \leq \infty$. Then $W\left(L^{p), \theta}, L^{p), \theta}\right)(\Omega)=L^{p), \theta}(\Omega)$.

Proof. According to the definition of the supremum, for an arbitrary $\eta>0$, there exists $0<\varepsilon_{0} \leq p-1$, such that for all $f \in L^{p), \theta}(\Omega)$,

$$
\sup _{0<\varepsilon \leq p-1}\left(\varepsilon^{\frac{\theta}{p-\varepsilon}}\|f\|_{p-\varepsilon}\right) \leq \varepsilon^{\frac{\theta}{p-\varepsilon_{0}}}\|f\|_{p-\varepsilon_{0}}+\eta
$$


Then we have

$$
\begin{aligned}
F_{f}^{p), \theta}(x) & =\left\|f \cdot \chi_{Q+x}\right\|_{p), \theta}=\sup _{0<\varepsilon \leq p-1}\left(\varepsilon^{\frac{\theta}{p-\varepsilon}}\left\|f \cdot \chi_{Q+x}\right\|_{p-\varepsilon}\right) \\
& \leq\left(\varepsilon_{0}^{\frac{\theta}{p-\varepsilon_{0}}}\left\|f \cdot \chi_{Q+x}\right\|_{p-\varepsilon_{0}}+\eta\right)=\varepsilon^{\frac{\theta}{p-\varepsilon_{0}}} F_{f}^{p-\varepsilon_{0}}+\eta
\end{aligned}
$$

and so

$$
F_{f}^{p), \theta}(x)=\sup _{0<\varepsilon \leq p-1}\left(\varepsilon^{\frac{\theta}{p-\varepsilon}}\left\|F_{f}\right\|_{p-\varepsilon}\right) \leq \varepsilon_{0}^{\frac{\theta}{p-\varepsilon_{0}}}\left\|F_{f}\right\|_{p-\varepsilon_{0}}+\eta .
$$

Thus from (10) and (11) we have

$$
\begin{aligned}
\|f\|_{W\left(L^{p), \theta}, L^{p), \theta}\right)} & =\left\|F_{f}^{p), \theta}\right\|_{p), \theta}=\sup _{0<\varepsilon \leq p-1} \varepsilon^{\frac{\theta}{p-\varepsilon}}\left\|F_{f}^{p), \theta}\right\|_{p-\varepsilon} \leq \varepsilon_{0}^{\frac{\theta}{p-\varepsilon_{0}}}\left\|F_{f}^{p), \theta}\right\|_{p-\varepsilon_{0}}+\eta \\
& \leq \varepsilon_{0}^{\frac{\theta}{p-\varepsilon_{0}}}\left\|\varepsilon_{0}^{\frac{\theta}{p-\varepsilon_{0}}}\right\| f \cdot \chi_{Q+x}\left\|_{p-\varepsilon_{0}}+\eta\right\|_{p-\varepsilon_{0}}+\eta \\
& \leq \varepsilon_{0}^{\frac{\theta}{p-\varepsilon_{0}}}\left\|\varepsilon_{0}^{\frac{\theta}{p-\varepsilon_{0}}}\right\| f \cdot \chi_{Q+x}\left\|_{p-\varepsilon_{0}}\right\|_{p-\varepsilon_{0}}+\varepsilon^{\frac{\theta}{p-\varepsilon_{0}}}\|\eta\|_{p-\varepsilon_{0}}+\eta \\
& =\varepsilon_{0}^{\frac{\theta}{p-\varepsilon_{0}}} \varepsilon_{0}^{\frac{\theta}{p-\varepsilon_{0}}}\|f\|_{W\left(L^{p-\varepsilon_{0}}, L^{p-\varepsilon_{0}}\right)}+\|\eta\|_{p-\varepsilon_{0}}+\eta \\
& =\varepsilon_{0}^{\frac{\theta}{p-\varepsilon_{0}}}\left(\varepsilon_{0}^{\frac{\theta}{p-\varepsilon_{0}}}\|f\|_{p-\varepsilon_{0}}\right)+\eta \cdot \mu(\Omega)+\eta .
\end{aligned}
$$

If $\eta \rightarrow 0$, the right side of (11) approaches to $C_{1}\|f\|_{W\left(L^{p), \theta}, L^{p), \theta}\right)}$ and we have

$$
\|f\|_{W\left(L^{p, \theta}, L^{p, \theta}\right)} \leq C_{1}\|f\|_{p), \theta}
$$

for some constant $C_{1}>0$.

Conversely let $f \in L^{p), \theta}$. For this $\eta>0$,we obtain

$$
\begin{aligned}
\|f\|_{p), \theta} & =\sup _{0<\varepsilon \leq p-1} \varepsilon^{\frac{\theta}{p-\varepsilon}}\|f\|_{p-\varepsilon} \leq \varepsilon_{0}^{\frac{\theta}{p-\varepsilon_{0}}}\|f\|_{p-\varepsilon_{0}}+\eta \\
& =\varepsilon_{0}^{\frac{\theta}{p-\varepsilon_{0}}}\|f\|_{W\left(L^{p-\varepsilon_{0}}, L^{p-\varepsilon_{0}}\right)}+\eta \leq \\
& =\varepsilon_{0}^{\frac{\theta}{p-\varepsilon_{0}}}\|\| f \cdot \chi_{Q+x}\left\|_{p-\varepsilon_{0}}\right\|_{p-\varepsilon_{0}}+\eta \\
& \leq\left\|\varepsilon_{0}^{\frac{\theta}{p-\varepsilon_{0}}}\right\| f \cdot \chi_{Q+x}\left\|_{p-\varepsilon_{0}}+\eta\right\|_{p-\varepsilon_{0}}+\eta \\
& \leq\left\|\varepsilon_{0}^{\frac{\theta}{p-\varepsilon_{0}}}\right\| f \cdot \chi_{Q+x}\left\|_{p-\varepsilon_{0}}\right\|_{p-\varepsilon_{0}}+\varepsilon_{0}^{\frac{\theta}{p-\varepsilon_{0}}}\|\eta\|_{p-\varepsilon_{0}}+\eta \\
& =\varepsilon_{0}^{\frac{-\theta}{p-\varepsilon_{0}}}\left(\varepsilon_{0}^{\frac{\theta}{p-\varepsilon_{0}}}\left\|\varepsilon_{0}^{\frac{\theta}{p-\varepsilon_{0}}}\right\| f \cdot \chi_{Q+x}\left\|_{p-\varepsilon_{0}}\right\| \|_{p-\varepsilon_{0}}\right)+\varepsilon_{0}^{\frac{\theta}{p-\varepsilon_{0}}}\|\eta\|_{p-\varepsilon_{0}}+\eta
\end{aligned}
$$

If $\eta \rightarrow 0$, then the right side of (12) approaches to $C_{2}\|f\|_{W\left(L^{p), \theta}, L^{p), \theta}\right)}$ and we have

$$
\|f\|_{p), \theta} \leq C_{2}\|f\|_{W\left(L^{p), \theta}, L^{p), \theta}\right)}
$$

for some constant $C_{2}>0$. Combining (12) and (13), we obtain $W\left(L^{p), \theta}, L^{p), \theta}\right)(\Omega)=$ $L^{p), \theta}(\Omega)$. 


\section{Hölder's inequality, Duality and reflexivity in grand Wiener amalgam spaces}

It is known by Theorem 2, that $\|\cdot\|_{W\left(L^{p), \theta}, L^{q), \theta}\right)}$ is a Banach function norm and $W\left(L^{p), \theta}, L^{q), \theta}\right)(\Omega)$ is a Banach function space.

Definition 2. The associate space of $W\left(L^{p), \theta}, L^{q), \theta}\right)(\Omega)$ determined by the associate norm $\|\cdot\|_{W\left(L^{p), \theta}, L^{q), \theta}\right)^{\prime}}$ is

$$
W\left(L^{p), \theta}, L^{q), \theta}\right)^{\prime}(\Omega)=\left\{g \in \mathcal{M}_{0}:\|g\|_{W\left(L^{p), \theta}, L^{q), \theta}\right)^{\prime}}<\infty\right\},
$$

where

$$
\|g\|_{W\left(L^{p), \theta}, L^{q), \theta}\right)^{\prime}}=\sup \left\{\int_{\Omega}|f g| d \mu: f \in \mathcal{M}_{0}(\Omega),\|f\|_{W\left(L^{p), \theta}, L^{q), \theta}\right)} \leq 1\right\} .
$$

THEOREM 4. (Hölder's inequality) If $f \in W\left(L^{p), \theta}, L^{q), \theta}\right)(\Omega)$ and $g \in$ $W\left(L^{p), \theta}, L^{q), \theta}\right)^{\prime}(\Omega)$, then $f g$ is integrable and

$$
\int_{\Omega} f(x) \cdot g(x) d x \leq\|f\|_{W\left(L^{p)}, L^{q)}\right)} \cdot\|g\|_{W\left(L^{p), \theta}, L^{q), \theta}\right)^{\prime}} .
$$

Proof. Since $W\left(L^{p), \theta}, L^{q), \theta}\right)(\Omega)$ is a Banach function space by Theorem 2, the proof is completed by Theorem 2.4., in [3] .

Proposition 6. The closure $\left.\overline{C_{0}^{\infty}}\right|_{W\left(L^{p), \theta}, L^{q), \theta}\right)}$ of the set $C_{0}^{\infty}$ in the space $W\left(L^{p), \theta}, L^{q), \theta}\right)(\Omega)$ consists of functions $f \in W\left(L^{p), \theta}, L^{q), \theta}\right)(\Omega)$ such that

$$
\lim _{\varepsilon \rightarrow 0} \varepsilon^{\frac{\theta}{p-\varepsilon}}\|f\|_{W\left(L^{p-\varepsilon}, L^{q-\varepsilon}\right)}=0
$$

where $C_{0}^{\infty}$ is the space of infinitely differentiable complex valued functions with compact support.

Proof. First we will show that (15) holds for $\left.\overline{C_{0}^{\infty}}\right|_{W\left(L^{p), \theta}, L^{q), \theta}\right)}$. Since $C_{0}^{\infty}(\Omega)$ is dense $L^{p}(\Omega)$, then $C_{0}^{\infty}(\Omega)$ is dense in $W\left(L^{p}, L^{q}\right)$ by Theorem 1, in [9] . Let $\left.f \in \overline{C_{0}^{\infty}}\right|_{W\left(L^{p), \theta}, L^{q), \theta}\right)}$. Then there exists a sequence $\left(f_{n}\right) \subset W\left(L^{p}, L^{q}\right)$ such that

$$
\left\|f_{n}-f\right\|_{W\left(L^{p}, L^{q}\right)} \rightarrow 0 .
$$

Thus for given $\eta>0$, there exists $n_{0} \in \mathbb{N}$ such that

$$
\left\|f_{n_{0}}-f\right\|_{W\left(L^{p}, L^{q}\right)}<\frac{\eta}{2}
$$

Since

$$
\varepsilon^{\frac{\theta}{p-\varepsilon}}\left\|f_{n_{0}}\right\|_{W\left(L^{p-\varepsilon}, L^{q-\varepsilon}\right)}=\varepsilon^{\frac{\theta}{p-\varepsilon}}\|\| f_{n_{0}} \chi_{Q+x}\left\|_{p-\varepsilon}\right\|_{q-\varepsilon},
$$

and $f_{n_{0}} \chi_{Q+x} \in L^{p}$, by the Hölder's inequality

$$
\varepsilon^{\frac{\theta}{p-\varepsilon}}\left\|f_{n_{0}} \chi_{Q+x}\right\|_{p-\varepsilon}=\varepsilon^{\frac{\theta}{p-\varepsilon}}\left\{\int_{\Omega}\left|f_{n_{0}}(t) \chi_{Q+x}\right|^{p-\varepsilon} d t\right\}^{\frac{1}{p-\varepsilon}}
$$




$$
\begin{aligned}
& \leq \varepsilon^{\frac{\theta}{p-\varepsilon}}\left\|f_{n_{0}} \chi_{Q+x}\right\|_{p} \mu(\Omega)^{\frac{1}{p-\varepsilon}-\frac{1}{p}} \\
& =\varepsilon^{\frac{\theta}{p-\varepsilon}} \mu(\Omega)^{\frac{\varepsilon}{p(p-\varepsilon)}}\left\|f_{n_{0}} \chi_{Q+x}\right\|_{p} .
\end{aligned}
$$

Thus from (17),

$$
\begin{aligned}
& \left\|\varepsilon^{\frac{\theta}{p-\varepsilon}}\right\| f_{n_{0}} \chi_{Q+x}\left\|_{p-\varepsilon}\right\|_{q-\varepsilon} \leq\left\|\varepsilon^{\frac{\theta}{p-\varepsilon}} \mu(\Omega)^{\frac{\varepsilon}{p(p-\varepsilon)}}\right\| f_{n_{0}} \chi_{Q+x}\left\|_{p}\right\|_{q-\varepsilon} \\
& =\varepsilon^{\frac{\theta}{p-\varepsilon}} \mu(\Omega)^{\frac{\varepsilon}{p(p-\varepsilon)}}\|\| f_{n_{0}} \chi_{Q+x}\left\|_{p}\right\|_{q-\varepsilon} \\
& \leq \varepsilon^{\frac{\theta}{p-\varepsilon}} \mu(\Omega)^{\frac{\varepsilon}{p(p-\varepsilon)}} \mu(\Omega)^{\frac{1}{q-\varepsilon}-\frac{1}{q}}\|\| f_{n_{0}} \chi_{Q+x}\left\|_{p}\right\|_{q} \\
& =\varepsilon^{\frac{\theta}{p-\varepsilon}} \mu(\Omega)^{\frac{\varepsilon}{p(p-\varepsilon)}+\frac{\varepsilon}{q(q-\varepsilon)}}\left\|f_{n_{0}}\right\|_{W\left(L^{p}, L^{q}\right)} \rightarrow 0,
\end{aligned}
$$

as $\varepsilon \rightarrow 0$. Hence there exists $\varepsilon_{0}$ such that when $\varepsilon<\varepsilon_{0}$,

$$
\varepsilon^{\frac{\theta}{p-\varepsilon}}\left\|f_{n_{0}}\right\|_{W\left(L^{p-\varepsilon}, L^{q-\varepsilon}\right)}=\left\|\varepsilon^{\frac{\theta}{p-\varepsilon}}\right\| f_{n_{0}} \chi_{Q+x}\left\|_{p-\varepsilon}\right\|_{q-\varepsilon} \leq \frac{\eta}{2} .
$$

Then by (16) and (18),

$$
\begin{aligned}
\varepsilon^{\frac{\theta}{p-\varepsilon}}\|f\|_{W\left(L^{p-\varepsilon}, L^{q-\varepsilon}\right)} & \leq \varepsilon^{\frac{\theta}{p-\varepsilon}}\left\|f_{n_{0}}-f\right\|_{W\left(L^{\left.p-\varepsilon, L^{q-\varepsilon}\right)}\right.}+\varepsilon^{\frac{\theta}{p-\varepsilon}}\left\|f_{n_{0}}\right\|_{W\left(L^{p-\varepsilon, L^{q-\varepsilon}}\right)} \\
& \leq\left\|f_{n_{0}}-f\right\|_{W\left(L^{p), \theta}, L^{q), \theta}\right)}+\frac{\eta}{2}<\frac{\eta}{2}+\frac{\eta}{2}=\eta
\end{aligned}
$$

when $\varepsilon<\varepsilon_{0}$. This completes the proof.

Proposition 7. If $q \leq p$, and $\theta \geq 1$, then the set $C_{0}^{\infty}(\Omega)$ is not dense in $W\left(L^{p), \theta}, L^{q), \theta}\right)(\Omega)$.

Proof. It is enough to give the proof for the special case $\Omega=(0,1)$. Let $f(t)=t^{\frac{1}{p}}$, for $p>1$. We showed in Proposition 2, that $t^{-\frac{1}{p}} \in W\left(L^{p), \theta}, L^{q), \theta}\right)(0,1)$. In the other hand since $\left.\left.\overline{C_{0}^{\infty}}\right|_{W\left(L^{p), \theta}, L^{q), \theta}\right)} \subset \overline{C_{0}^{\infty}}\right|_{L^{p), \theta}}$ and $\left.t^{-\frac{1}{p}} \notin \overline{C_{0}^{\infty}}(0,1)\right|_{L^{p, \theta},}$,

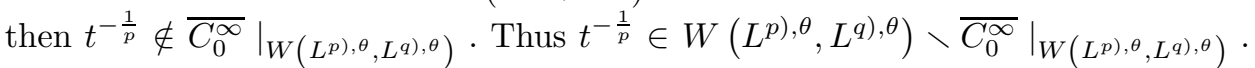
This ends the proof.

Definition 3. Let $\left(X,\|\cdot\|_{X}\right)$ be a Banach function space and let $f \in X$ be any arbitrary function. We say that $f$ has absolutely continuous norm in $X$ if

$$
\lim _{n \rightarrow \infty}\left\|f \chi_{E_{n}}\right\|_{X}=0
$$

for every sequence $\left\{E_{n}\right\}_{n \in \mathbb{N}}$ satisfying $E_{n} \rightarrow \phi$. We will denote the subspace of the functions in $X$ with absolutely continuous norm by $X_{a}$. If $X=X_{a}$, then $X$ it self is said to have absolute continuous norm.

We need the following known two theorems to find the dual of the grand Wiener amalgam spaces..

Theorem 5. ( See [3], Corollary 4.3). The dual space $X^{*}$ of a Banach function space $X$ is isometric to the associate space $X^{\prime}$ if and only if $X$ has absolutely continuous norm.

Theorem 6. ( See [3], Corollary 4.4). A Banach function space is reflexive if and only if both $X$ and its associate space $X^{\prime}$ have absolute continuous norm. 
THEOREM 7. The grand Wiener amalgam spaces $W\left(L^{p), \theta}, L^{q), \theta}\right)(c, d)$ has not absolute continuous norm.

Proof. We will give the proof of this theorem for the interval $(c, d)=(0,1)$. One can prove similarly this theorem for any interval $(c, d)$. Take the function $f(t)=t^{-\frac{1}{p}}$. We will show that $f$ is not absolute continuous. Let $Q=(0, a) \subset(0,1)$. Then

$$
\begin{aligned}
& \left\|\left(t^{-\frac{1}{p}} \chi_{Q}\right) \chi_{Q+x}\right\|_{p), \theta}=\left\|t^{-\frac{1}{p}} \chi_{Q \cap Q+x}\right\|_{p), \theta}=\left\|t^{-\frac{1}{p}} \chi_{(x, a)}\right\|_{p), \theta} \\
= & \sup _{0<\varepsilon \leq p-1} \varepsilon^{\frac{\theta}{p-\varepsilon}}\left\|t^{-\frac{1}{p}} \chi_{(x, a)}\right\|_{p-\varepsilon}=\sup _{0<\varepsilon \leq p-1} \varepsilon^{\frac{\theta}{p-\varepsilon}}\left(\int_{x}^{a}\left|t^{-\frac{1}{p}}\right|^{p-\varepsilon}\right)^{\frac{1}{p-\varepsilon}} \\
= & \sup _{0<\varepsilon \leq p-1} \varepsilon^{\frac{\theta}{p-\varepsilon}}\left(\int_{x}^{a} t^{-\frac{p-\varepsilon}{p}}\right)^{\frac{1}{p-\varepsilon}}=\sup _{0<\varepsilon \leq p-1} \varepsilon^{\frac{\theta}{p-\varepsilon}}\left(\int_{x}^{a} t^{-1+\frac{\varepsilon}{p}}\right)^{\frac{1}{p-\varepsilon}} \\
= & \sup _{0<\varepsilon \leq p-1} \varepsilon^{\frac{\theta}{p-\varepsilon}}\left(\frac{p}{\varepsilon}\left[a^{\frac{\varepsilon}{p}}-x^{\frac{\varepsilon}{p}}\right]\right)^{\frac{1}{p-\varepsilon}}=\sup _{0<\varepsilon \leq p-1} \varepsilon^{\frac{\theta-1}{p-\varepsilon}} p^{\frac{1}{p-\varepsilon}}\left[a^{\frac{\varepsilon}{p}}-x^{\frac{\varepsilon}{p}}\right]^{\frac{1}{p-\varepsilon}} .
\end{aligned}
$$

It is easy to see that $\varepsilon^{\frac{\theta-1}{p-\varepsilon}}$ and $p^{\frac{1}{p-\varepsilon}}$ are increasing functions of $\varepsilon$. Since $0<a<1$, from (19) we have

$$
\left\|\left(t^{-\frac{1}{p}} \chi_{Q}\right) \chi_{Q+x}\right\|_{p), \theta}=(p-1)^{\theta-1} p\left[1-x^{\frac{p-1}{p}}\right] .
$$

Also since $x$ tends to 0 as $a \rightarrow 0$, from (20) we obtain

$$
\begin{aligned}
\lim _{a \rightarrow 0}\left\|t^{-\frac{1}{p}} \chi_{(0, a)}\right\|_{W\left(L^{p), \theta}, L^{q), \theta}\right)} & =\lim _{a \rightarrow 0}\|\| t^{-\frac{1}{p}} \chi_{(x, a)}\left\|_{p), \theta}\right\|_{q), \theta} \\
& =\lim _{a \rightarrow 0}\left\|(p-1)^{\theta-1} p\left[1-x^{\frac{p-1}{p}}\right]\right\|_{q), \theta} \\
& =\left\|\lim _{a \rightarrow 0}(p-1)^{\theta-1} p\left[1-x^{\frac{p-1}{p}}\right]\right\|_{q), \theta} \\
& =(p-1)^{\theta-1} p\left\|\lim _{x \rightarrow 0}\left(1-x^{\frac{p-1}{p}}\right)\right\|_{q), \theta} \\
& =(p-1)^{\theta-1} p\|1\|_{q), \theta} \neq 0 .
\end{aligned}
$$

Thus $f(t)=t^{-\frac{1}{p}} \in W\left(L^{p), \theta}, L^{q), \theta}\right)(0,1)$, but it has not absolute continuous norm in $W\left(L^{p), \theta}, L^{q), \theta}\right)(0,1)$. Then by definition $3, W\left(L^{p), \theta}, L^{q), \theta}\right)(0,1)$ has not absolute continuous norm.

Corollary 2. The grand Wiener amalgam spaces $W\left(L^{p), \theta}, L^{q), \theta}\right)(c, d)$ is not reflexive.

Proof. The proof is clear from Theorem 9 and Theorem 10.

Corollary 3. The dual space $\left(W\left(L^{p), \theta}, L^{q), \theta}\right)(c, d)\right)^{*}$ of the grand Wiener amalgam space $W\left(L^{p), \theta}, L^{q), \theta}\right)(c, d)$ is not isometric to the associate space $\left(W\left(L^{p), \theta}, L^{q), \theta}\right)(c, d)\right)^{\prime}$ of this grand Wiener amalgam space $W\left(L^{p), \theta}, L^{q), \theta}\right)(c, d)$.

Proof. The proof of this theorem is easy from Theorem 8 and Theorem 10. 
TheOREM 8. Let $\frac{p}{p-1}=p^{\prime}, \frac{q}{q-1}=q^{\prime}$ and $1<p, q<\infty$. Then

$$
\left(W\left(L^{p), \theta}, L^{q), \theta}\right)(\Omega)\right)^{\prime}=W\left(L^{p)^{\prime}, \theta}, L^{q)^{\prime}, \theta}\right)(\Omega)
$$

and the norms $\|g\|_{W\left(L^{\left.p)^{\prime}, L^{q}\right)^{\prime}}\right)}$ and

$$
\|g\|_{W\left(L^{p), \theta}, L^{q), \theta}\right)^{\prime}}=\left\{\begin{array}{c}
\sup \int_{\Omega}|f(x) g(x)| d x: f \in W\left(L^{p), \theta}, L^{q), \theta}\right)(\Omega), \\
\|f\|_{W\left(L^{p)}, L^{q)}\right)} \leq 1
\end{array}\right\}
$$

are equivalent.

Proof. If $f \in W\left(L^{p), \theta}, L^{q), \theta}\right)(\Omega)$ and $g \in W\left(L^{p)^{\prime}, \theta}, L^{q)^{\prime}, \theta}\right)(\Omega)$, then

$$
\|f\|_{W\left(L^{p), \theta}, L^{q), \theta}\right)} \cdot\|g\|_{W\left(L^{p)^{\prime}, \theta}, L^{q)^{\prime}, \theta}\right)}=\|\| f \cdot \chi_{Q+x}\left\|_{p), \theta}\right\|_{q), \theta}\|\| g \cdot \chi_{Q+x}\left\|_{p)^{\prime}, \theta}\right\|_{q)^{\prime}, \theta} \cdot
$$

Since $\left\|f \cdot \chi_{Q+x}\right\|_{p), \theta} \in L^{q), \theta}$ and $\left\|g \cdot \chi_{Q+x}\right\|_{L^{p)^{\prime}, \theta}} \in L^{q)^{\prime}, \theta}$, by the Hölder inequality for the generalized grand Lebesgue space $\left\|f \cdot \chi_{Q+x}\right\|_{p), \theta}\left\|g \cdot \chi_{Q+x}\right\|_{L^{p)^{\prime}, \theta}} \in L^{1}(\Omega)$ and

$$
\|\| f \cdot \chi_{Q+x}\left\|_{p), \theta}\right\| g \cdot \chi_{Q+x}\left\|_{p)^{\prime}, \theta}\right\|_{1} \leq\|\| f \cdot \chi_{Q+x}\left\|_{p), \theta}\right\|_{q), \theta}\|\| g \cdot \chi_{Q+x}\left\|_{p)^{\prime}, \theta}\right\|_{q)^{\prime}, \theta} \cdot
$$

Also since $f \cdot \chi_{Q+x} \in L^{p), \theta}$ and $g \cdot \chi_{Q+x} \in L^{p)^{\prime}, \theta}$, one more applying the Hölder inequality for the generalized Lebesgue space we have

$$
\begin{aligned}
\|f g\|_{1}= & \|f g\|_{W\left(L^{1}, L^{1}\right)}=\|\|\left(f \cdot \chi_{Q+x}\right)\left(g \cdot \chi_{Q+x}\right)\left\|_{1}\right\|_{1} \\
& \leq\|\| f \cdot \chi_{Q+x}\left\|_{p), \theta}\right\| g \cdot \chi_{Q+x}\left\|_{L^{p)^{\prime}, \theta}}\right\|_{1} .
\end{aligned}
$$

Combining (21) and (22) we obtain

$$
\int_{\Omega}|f(x) \cdot g(x) d x|=\|f \cdot g\|_{1} \leq\|f\|_{W\left(L^{p)}, L^{q)}\right)} \cdot\|g\|_{W\left(L^{\left.p)^{\prime}, L^{q}\right)^{\prime}}\right)} .
$$

From this inequality we have

$$
\|g\|_{W\left(L^{p), \theta}, L^{q), \theta}\right)^{\prime}}=\left\{\begin{array}{c}
\sup \int_{\Omega}|f(x) g(x)| d x: f \in W\left(L^{p), \theta}, L^{q), \theta}\right)(\Omega), \\
\|f\|_{W\left(L^{p)}, L^{q)}\right)} \leq 1
\end{array}\right\} \leq\|g\|_{W\left(L^{\left.p)^{\prime}, L^{q}\right)^{\prime}}\right)} .
$$

This implies

$$
W\left(L^{p)^{\prime}, \theta}, L^{q)^{\prime}, \theta}\right)(\Omega) \subset\left(W\left(L^{p), \theta}, L^{q), \theta}\right)(\Omega)\right)^{\prime}
$$

If one uses the same technic in $([13]$, Theorem 11.7.1 $(c))$, obtains

$$
W\left(L^{p)^{\prime}, \theta}, L^{q)^{\prime}, \theta}\right)(\Omega) \supset\left(W\left(L^{p), \theta}, L^{q), \theta}\right)(\Omega)\right)^{\prime} .
$$

Thus we have

$$
W\left(L^{p)^{\prime}, \theta}, L^{q)^{\prime}, \theta}\right)(\Omega)=\left(W\left(L^{p), \theta}, L^{q), \theta}\right)(\Omega)\right)^{\prime}
$$


Since the spaces $W\left(L^{p)^{\prime}, \theta}, L^{q)^{\prime}, \theta}\right)(\Omega)$ and $\left(W\left(L^{p), \theta}, L^{q), \theta}\right)(\Omega)\right)^{\prime}$ are equal and they are Banach spaces with respect to the norms $\|\cdot\|_{W\left(L^{\left.p)^{\prime}, L^{q}\right)^{\prime}}\right)}$ and

$\|g\|_{W\left(L^{p), \theta}, L^{q), \theta}\right)^{\prime}}=\left\{\sup \int_{\Omega}|f(x) g(x)| d x: f \in W\left(L^{p), \theta}, L^{q), \theta}\right)(\Omega),\|f\|_{W\left(L^{p)}, L^{q)}\right)} \leq 1\right\}$

respectively, then these norms are equivalent by Two norm theorem (see Theorem $7.3 .3,[19])$

\section{References}

[1] Anatriello G, Chil R and Fiorenza A. Identification of fully Measurable Grand Lebesgue Spaces. Journal of Function Spaces, Vol. 2017.

[2] Anatriello G. Iterated grand and small Lebesgue spaces. Collect. Math. 2014;64:273 - 284.

[3] Bennett C and Sharpley, R. Interpolation of Operators, Academic Press, INC, Orlando, Florida,1988.

[4] Capone C, Formica MR, Giova R. Grand Lebesgue spaces with respect to measurable functions. Nonlinear Analysis 2013; $85: 125-131$.

[5] Capone C, and Fiorenza A. On small Lebesgue spaces. Journal of function spaces and applications $2005 ; 3(1): 73-89$.

[6] Castillo R. E, Raferio H. Inequalities with conjugate exponents in grand Lebesgue spaces. Hacettepe Journal of Mathematics and Statistics 2015;44 (1) : 33 - 39.

[7] Castillo R. E, Raferio H. An Introductory Course in Lebesgue Spaces. Springer International Publishing Switzerland 2016.

[8] Danelia N, Kokilashvili V. On the approximation of periodic functions within the frame of grand Lebesgue spaces. Bulletin of the Georgian national academy of sciences 2012;6(2) : $11-16$.

[9] Feichtinger HG, Banach convolution algebras of Wiener's type, Proc. Conf. "Functions, Series, Operators", Budapest, 1980, Colloquia Math. Soc. J. Bolyai, North Holland Publ. Co., Amsterdam- Oxford- New York $1983: 509$ - 524.

[10] Feichtinger HG and Gröchenig KH, Banach spaces related to integrable group representations and their atomic decompositions I, J. Funct. Anal., 86(1989), 307-340.

[11] Fiorenza A, and Karadzhov GE. Grand and small Lebesgue spaces and their analogs, Journal for Analysis and its Applications 2004; 23 (4) : 657 - 681

[12] Fiorenza A. Duality and reflexity in grand Lebesgue spaces, Collect. Math. 2000; 51 (2) : $131-148$.

[13] Greco L, Iwaniec T, Sbordone C. Inverting the p-harmonic operator, Manuscripta Math.1997; $92: 259-272$.

[14] Gürkanlı AT, Inclusion and the approximate identities of the generalized grand Lebesgue spaces. Turk J Math Doi:10.3906/mat-1803-89, (Accepted for publication)

[15] Heil C, An Introduction to Weighted Wiener Amalgams, In: Wavelets and their Applications (Chennai, 2002), Allied Publishers, New Delhi, 2003 : $183-216$.

[16] Holland F. Square -summable positive-definite functions on real line, Linear operators Approx. II, Ser. Numer. Math. 25, Birkhauser, Basel, 1974, $247-257$.

[17] Holland F. Harmonic analysis on amalgams of $L^{p}$ and $\ell^{q}$, London Math. Soc. $10-\mathbf{2}$, (1975), $295-305$.

[18] Iwaniec T, Sbordone C. On the integrability of the Jacobian under minimal hypotheses, Arc. Rational Mech. Anal. 1992; 119 : 129 - 143..

[19] Larsen R, Functional Analysis an Introduction, Marcel Dekker, INC. New York, 1973.

Istanbul Arel University Faculty of Science and Letters Department of MatheMATICS AND COMPUTER SCIENCES

E-mail address: turangurkanli@arel.edu.tr 